\title{
A network model of retrieval failures: Moving forward with incorporating clinical data
}

\author{
Nichol Castro
}

Department of Communicative Disorders and Sciences, University at Buffalo

\author{
Author Note
}

Nichol Castro (iD) https://orcid.org/0000-0001-8691-7968

Correspondence concerning this article should be addressed to Nichol Castro, Department of Communicative Disorders and Sciences, 3435 Main Street, Cary Hall Rm 122, University at Buffalo, Buffalo, NY 14215. Email: nicholca@buffalo.edu 


\begin{abstract}
Understanding retrieval failures requires a cognitive model that considers not just impaired processes, but also the role of structure. The development of a network model of retrieval failures requires the inclusion of clinical data, but there remain methodological issues in using and interpreting such data: locus of retrieval failure, heterogeneity of individuals, and progression of disorder/disease. Techniques from network science may prove useful in addressing these issues, while capturing the complexity of language disorders. Critically, any network model we employ could have downstream impact on clinical practice, which ultimately impacts patient lives, harkening the need for theoretically well-informed network models.
\end{abstract}

Keywords: network science, word retrieval, aphasia, dementia, mental lexicon 


\section{Introduction}

For most, retrieving information from memory occurs without difficulty. Information is retrieved quickly and efficiently. However, experiences of memory retrieval failures occur in both typical adults and adults with cognitive or language impairments. One area of continued investigation is understanding how memory retrieval breaks down differently in typical and clinical populations, not only for our understanding of human cognition and those disorders, but also for practical implications, like identifying and treating individuals with clinically significant memory retrieval impairments.

For a specific example, this paper will focus on word retrieval failures. Word retrieval from memory during spoken language discourse often occurs without issue. However, we all experience tip-of-the-tongue states (TOTs), instances where a person knows a word but is unable to produce it in the moment, and other speech errors. While TOTs are a common phenomenon across individuals, these word retrieval failures increase in frequency with normal aging (Burke et al., 1991; Shafto et al., 2007) and can be a feature of language impairment in dementia and aphasia (e.g., Beeson et al., 1997). Knowing which TOTs are "normal" from those that are potential indicators of a cognitive or language impairment is critical for early intervention.

While prominent models of language have identified the processes used in word retrieval and how those processes break down when word retrieval fails, there has been a growing body of research highlighting the importance of considering the role of mental lexicon structure on word retrieval processes. That is, the way in which words are organized in memory has implications for how individuals retrieve those words. Focusing on the structure of words in memory and the interaction of structure and process has been propelled by recent application of network science to the cognitive sciences (Castro \& Siew, 2020), an emerging area called cognitive network 
science (Baronchelli et al., 2013; Siew et al., 2019). In the study of word retrieval, the cognitive network science lens has been used to model the interaction of word-word similarity relationships in memory and word retrieval. This approach has been inspired by prominent language models, including Dell's Interactive Activation model (Dell, 1986; Dell \& O'Seaghdha, 1992), with a key difference in focusing on how each pair of words in a person's mental lexicon are related (or not) to each other semantically, phonologically, orthographically, and so on. Current models of language are hard pressed to make explicit predictions of structural influence on process. Therefore, validating a model of retrieval failures using principles from network science is necessary to explain the accumulating evidence highlighting the interaction of mental lexicon structure on word retrieval.

Because word retrieval failures tend to be infrequent in typical adults, modeling word retrieval and its failures has benefited from the study of clinical populations, including persons with aphasia and dementia. Aphasia is a language disorder often resulting from a left hemisphere stroke and presents with significant word retrieval failures (called anomia). Dementia refers broadly to neurodegenerative diseases, many of which present with word retrieval difficulties. For example, semantic dementia is a variant of frontotemporal dementia characterized by the degradation of semantic memory, resulting in difficulty with word retrieval.

As the cognitive network science lens continues to focus on developing and validating a model of retrieval failures, the inclusion of data from clinical populations will continue (for a review, see Kenett \& Faust, 2019). While there are many open questions that need to be addressed in a network model of retrieval failures, this paper serves to provide a brief commentary on methodological issues that are exacerbated in the study of clinical data, namely the locus of retrieval failure, heterogeneity of individuals, and progression of disorder/disease. 
First, there will be a brief review of how clinical picture naming data has been used to develop one prominent language model, Dell's Interactive Activation (IA) model (Dell, 1986; Dell \& O’Seaghdha, 1992),, and a novel network model of retrieval failures (Castro et al., 2020). Then, a discussion will focus on how the cognitive network science lens could be used to address methodological issues in the use of clinical data during model development. It should be noted that this paper is not intended to directly compare the strengths and weaknesses of the IA model and a network model of retrieval failures. Instead, the IA model should be viewed by cognitive network scientists and others as an example of how to successfully develop a sophisticated model with theoretical and clinical importance.

\section{Modeling Retrieval Failures}

Cognitive models are dependent on the type of data used to develop them. In the study of word retrieval, there are two primary models: serial models developed based on chronometric data and interactive models developed based on speech error data (Levelt, 1999). Both types of models have provided valuable insight into the structure and processes of language. However, both models are also limited in the set of questions that can be asked. For example, because the IA model was developed with a focus on speech error data (Dell, 1986; Dell \& O’Seaghdha, 1992), this model has been successful to the study of word retrieval failures in persons with aphasia and typical adults (e.g., Dell et al., 1997; Foygel \& Dell, 2000; Martin \& Dell, 2019; Schwartz et al., 2006). Modeling retrieval failures using a cognitive network science lens has also relied on the use of speech error data (e.g., Casstro \& Stella, 2019; Castro et al., 2020; Vitevitch \& Castro, 2015). This section will briefly review the IA model and a network model in its application to studying word retrieval failures.

\subsection{The Interactive Activation model}


From a structural standpoint, the IA model represents linguistic units, or pieces of information, as nodes (see Figure 1A; Dell \& O'Seaghdha, 1992). Specifically, these nodes represent three systems in the model: semantic features, lemmas (or words), and phonemes. Bidirectional connections in the model link semantic features to lemmas and lemmas to phonemes. There are no links between nodes in the same system (e.g., between lemmas).

Word retrieval in this model occurs through two stages using a mechanism of spreading activation: lemma access and phonological access (Dell \& O’Seaghdha, 1992; Schwartz et al., 2006). Lemma access first begins when semantic feature nodes corresponding to the intended word (e.g., the features identified in a picture of the to-be-named word) receive a jolt of activation. That activation then spreads through the network interactively (i.e., top-down: semantics to phonology; bottom-up: phonology to semantics). During spreading activation, a variety of lemmas may receive some activation depending on their connections within the network. The spread of activation stops when the most active lemma is selected, ideally corresponding to the intended word. After lemma selection, the phonological access stage begins by providing a jolt of activation to the selected lemma. Activation spreads again through the network, with activation settling on the most active phoneme nodes, ideally corresponding to the selected lemma.

The key feature of the IA model is the interactivity that occurs within the network during both stages of word retrieval (Schwartz et al., 2006). This feature allows phonological information to influence lemma selection and allows semantic information to influence phonological retrieval. However, it should be noted that the two separate stages limit the influence of interactivity at the second stage. Regardless, this interactivity is crucial to explaining why certain speech errors emerge. 
To model speech error production, Dell and colleagues "lesioned" certain parameters of the IA model. Two models of "lesions" were proposed by Dell and colleagues: weight-decay model (Dell et al., 1997) and semantic-phonological model (Foygel \& Dell, 2000). In the weightdecay model (Dell et al., 1997), the parameters of weight (i.e., the strength of connections in the model, which determine how much activation spreads across a connection) and decay (i.e., the rate of activation decay in the model) were manipulated. Simulations were then conducted to obtain error profiles, or the number of different speech error types, for a particular set of parameter lesions. The simulation results indicated that "weight-lesions" tended to produce more nonword errors, while "decay-lesions" tended to produce more semantic and mixed errors. Furthermore, these simulated lesions provided remarkably similar error profiles to error profiles of persons with aphasia, providing a way to describe an individual's naming impairment (Dell et al., 1997). For example, an individual who produces many nonword errors may have difficulty with the transmission (as opposed to maintenance) of activation in their mental lexicon.

In the semantic-phonological model (Foygel \& Dell, 2000), the decay parameter was dropped and instead the model "lesioned" either the weights of semantic connections (i.e., connections between semantic and lemma nodes) or phonological connections (i.e., connections between lemma and phonological nodes). Again, simulations were conducted to obtain error profiles for different lesion parameter sets and compared to the results of the weight-decay model. The simulations indicated that semantic lesions corresponded with the decay lesions of the prior model and phonological lesions corresponded with the weight-lesions of the prior model. While the outcomes of the two models were similar, the semantic-phonological model in comparison to the weight-decay model was better at accounting for repetition data, lexical or phonological errors, and connecting a patient's model characterization to diagnostic category. 
Furthermore, this model has led to novel variables in describing the linguistic profile of a person with aphasia, namely s- and p- weights (Foygel \& Dell, 2000). Such variables can be used to identify individuals who may have more difficulty with semantic processing (low s-weights) or phonological processing (low p-weights).

The IA model is now being used to develop innovative treatments for persons with aphasia that focus on impaired spreading activation processes. For example, Martin and Dell (2019) show that some persons with aphasia have a deficit in the transmission of activation, the maintenance of activation, or both. Using a delayed $(5 \mathrm{sec})$ picture naming task, they found that individuals who did better after the delay mapped onto the IA model when the weight parameter was lesioned, reflecting that more processing time allowed activation to transmit and settle on the correct lemma. On the other hand, individuals who did worse after the delay mapped onto the IA model when the decay parameter was lesioned, reflecting that activation could not be maintained on the correct lemma for long enough (Martin \& Dell, 2019). Knowing how spreading activation processes are impaired for a given individual could help clinicians to target treatment to those specific impairments. Thus, the IA model is an exemplar for how to develop not just a theoretically important model, but a model that also can inform clinical practice.

\subsection{Networks of Words}

Prominent language models tend to focus on the processes of language as they occur within certain linguistic knowledge domains (e.g., semantics and phonology). These models often verbally describe the structure of linguistic knowledge (e.g., semantic features shared between semantically similar words), but few make explicit predictions of how that structure influences language processes. Some prominent language models have been able to account for aspects of "local" similarity between words; for example, neighborhood effects, or the number of 
similar words of a target word, have been well documented in the phonological and semantic literatures (e.g., Mirman \& Magnuson, 2008; Vitevitch \& Luce, 2016), including in the study of persons with aphasia (e.g., Gordon, 2002; Middleton \& Schwartz, 2010). However, prominent models of language have not been able to account for more large-scale similarity relationships between words, in part because they do not model lexicons of such a large size (e.g., six lemmas in the IA model; Dell et al., 1997; Foygel \& Dell, 2000). That is, how are the tens of thousands of words a person knows related to each other, and how does that very large and complex structure influence language processes, like word retrieval?

The growing application of network science to study cognitive systems has led to an increased re-exploration in understanding the role of mental lexicon structure on language processes (Castro \& Siew, 2020). Many recent applications focus on word-word similarity networks, or Networks of Words (see Figure 1B). Specifically, nodes in the network represent words or concepts and links are placed between nodes based on a definition of similarity (e.g., semantic or phonological similarity). One could view a Network of Words as a flattening of the tripartite network of the IA model. These word-word similarity networks may use participantdriven data to determine similarity, as done with free association networks (i.e., participants produce the first word that comes to mind in response to a cue word), as well as based on dictionaries, thesauri, and other linguistic corpora (e.g., feature norms). For example, phonological and orthographic networks place links between words if they differ by a certain number of phonemes/letters (e.g., one phoneme/letter difference through addition, substitution, or deletion). This approach has shown that greater consideration of the structure of words in memory is necessary for a complete understanding of a variety of language processes (e.g., Beckage \& Colunga, 2015; Vitevitch, 2008; Vitevitch et al., 2014), including word retrieval 
(Castro \& Stella, 2019; Castro et al., 2020; Chan \& Vitevitch, 2010; Vitevitch \& Castro, 2015; Vitevitch, Chan, \& Goldstein, 2014; Vitevitch \& Sommers, 2003).

Relevant to the study of word retrieval failures in persons with aphasia, a recent multiplex lexical network has been proposed (Castro \& Stella, 2019; Castro et al., 2020). From a structural standpoint, a multiplex network is one with several layers of networks connected to each other through a similar set of nodes. In this case, the same words are represented as nodes on each of four layers, with different types of similarity connections between nodes on each layer. Specifically, there are three layers representing semantic similarity between words, namely though free association, synonyms, and generalizations, and one layer representing phonological similarity between words. Inherent in this approach is the assumption that the underlying structure of word-word similarity relationships captures emergent information about word representations in memory (e.g., words belonging to the same semantic category would be more closely connected to each other than to words in different categories in a semantic network), which has repercussions for processing that occurs within that network structure (e.g., the structure of the network itself determines where activation spreads; Castro et al., 2020).

Castro et al. (2020) found two network measures that influenced successful word retrieval and speech errors on a picture naming task in persons with aphasia: closeness centrality and degree (see also Castro \& Stella, 2019). Closeness centrality is a network measure that captures how well-connected a word is to all others in the network, such that nodes with high closeness centrality tend to reside on short-cut paths in the network that link otherwise distantly connected nodes, whereas nodes with low closeness centrality tend to reside on the periphery of a network. The authors suggested that words with high closeness centrality tend to "pop out" in the network, allowing them to be more easily retrieved. Network degree (akin to neighborhood 
density in the psycholinguistic literature) captures the number of immediately connected nodes of a given node, such that high degree nodes are connected to many immediate nodes, whereas low degree nodes are connected to few immediate nodes. The authors suggested that words with high degree had multiple sources of converging evidence across different linguistic systems (semantics and phonology), allowing them to be more easily retrieved, but on incorrect trials this overlap of semantic and phonological influence resulted in a greater likelihood of producing mixed errors. Notably, such structural measures of a word in the mental lexicon, and their influence on processing, are not readily computed from existing language models.

By employing principles of spreading activation, this recent multiplex lexical network provided a verbal account for how the network structure of word-word similarity relationships influenced language processes (Castro et al., 2020). Simple simulations of such a verbal account have ensued in other literature using Networks of Words, with similar model performance as behavioral data with few parameters manipulated (Siew, 2019; Vitevitch et al., 2011; Vitevitch $\&$ Storkel, 2013). The verbal account of Castro et al (2020) remains to be validated with simulations and additional behavioral data from typical adults and persons with aphasia, but this verbal model has the potential to provide a new perspective on the structural influence of word representations in memory on word retrieval processes, and ultimately could inform clinical approaches to diagnostics and treatment.

\section{Developing a Network Model of Retrieval Failures: Methodological Considerations with the use of Clinical Data}

There are many open areas of investigation in continued development of a network model of retrieval failures. A common question asked of cognitive network scientists is how best to define the nodes and links in the network of interest. In the case of the multiplex lexical network, 
researchers must determine which, and how many, aspects of semantic and phonological similarity need to be included. Another issue that needs to be addressed is determining the process, and its parameters, that underlie the phenomenon of interest. In the case of the multiplex lexical network, a simple spreading activation process was verbally described, but other process mechanisms need to be considered in conjunction with or in replace of spreading activation. While these are critical, foundational issues in model development, this paper will focus on methodological issues in interpreting and using clinical data for model development, namely the locus of retrieval failure, heterogeneity of individuals, and progression of disorder/disease. Importantly, the cognitive network science approach to the study of word retrieval may be wellsuited to address these long-standing methodological issues in ongoing development of a network model of retrieval failures.

\subsection{Locus of retrieval failure: Disentangling structure and process}

Models of language describe how words are represented in memory and a process(es) that is used to search for, select, and extract words from memory. In theory, these aspects, structure and process, of language models are separable. However, a person's mental lexicon is not observable without employing a process to extract it. For example, while researchers often use vocabulary tests to estimate the size of a person's lexicon, those vocabulary tests require that an individual be able to access and extract information from their lexicon. Performance on the vocabulary test, although often interpreted as reflecting the contents of a person's lexicon, could simply reflect their processing abilities or the product of an interaction of structure and process. This is especially important to consider when using data from clinical populations who may have known processing-related impairments, thus muddying the interpretation of any structural difference observed in a network. 
Different clinical populations have different loci of word retrieval impairment. Mirman and Britt (2014) provide common behavioral evidence of structure-based impairments (which they called storage deficits) and process-based impairments (which they called access deficits) that distinguish the locus of words retrieval impairments between persons with semantic dementia and persons with aphasia, respectively. For example, aphasia has long been described as a process impairment (McNeil, 1982; McNeil \& Pratt, 2001). Evidence supporting the notion that aphasia (in contrast to semantic dementia) results in impaired processes includes sensitivity to cueing, sensitivity to presentation rate, performance inconsistency, negative serial position effect, sensitivity to number and strength of competitors, combined negative effects of serial position and competitors, disordered activation of strong and weak competitors, correlation with executive function deficits, and reduced frequency effects (Mirman \& Britt, 2014).

Knowing the locus of word retrieval impairments is clinically important, especially in the context of treatment. If the language network is degrading structurally as in semantic dementia, interventions might need to focus on how to make existing words resilient (for a review, see Henry et al., 2008). For example, this may require strengthening existing connections between words and adding in new or previously lost connections. On the other hand, if the language network is intact but processes are impaired as in aphasia, interventions might need to focus on strengthening cognitive skills needed for word retrieval (e.g., Dignam et al., 2017). For example, given impaired spreading activation processes in persons with aphasia, treating verbal shortterm memory may be efficacious (Martin \& Dell, 2019).

Disentangling structure and process may not be methodologically feasible, nor may it be a necessity, at least in the network science framework. Because the network science framework makes a conscious assumption that structure interacts with process, researchers can more closely 
consider the role of structure on process with the computational and mathematical tools of network science. As described previously, this was the approach of Castro et al. (2020) who identified specific network structure measures that influenced picture naming by persons with aphasia. While their study only provided a verbal account of how structure and process interacted, simulations conducted on the proposed language network could be used to further substantiate these claims. For instance, ongoing work in our lab is comparing simulations that degrade spreading activation in the network (akin to the work by Dell and colleagues) to simulations that remove connections in the network (reflecting a degraded structure), and compares those simulation outputs to the picture naming data of different clinical populations to assess which set of simulations better predicts the behavioral data. Critically, the network science framework allows for a way to test clear predictions about the interaction of mental lexicon structure and word retrieval processes for a variety of clinical populations, and provides a mechanism for considering different loci of word retrieval failure.

\subsection{Heterogeneity of individuals: Moving away from group-level networks}

It is well-known that there is significant heterogeneity between individuals within a given clinical diagnosis. No two brains are identical, and even the etiology of the same disease or stroke do not result in identical outcomes. Classical approaches in clinical aphasiology, for example, have resulted in broad diagnostic categories, like Broca's and Wernicke's aphasia, as well as broad fluency distinctions (i.e., fluent vs nonfluent aphasia), that are still commonly employed today. However, modern approaches to studying aphasia, which take into consideration the findings of cognitive and computational models (e.g., IA model), emphasize that the heterogeneity between persons with aphasia within the same diagnostic category requires a more comprehensive assessment of an individual's strengths and weaknesses (Caramazza, 
1984; Clough \& Gordon, 2020; Tremblay \& Dick, 2016). It is no longer enough to say that a person has a certain type of aphasia. Instead, researchers are moving towards more wholistic cognitive-linguistic profiles to describe the abilities of a person with aphasia.

Along with this recognition of between-subject heterogeneity, there has been greater emphasis on conducting case series studies (as opposed to patient group studies), in conjunction with single-subject studies (Schwartz \& Dell, 2010). Case series studies examine a select number of individuals (e.g., $N=10$ ) who are expected to vary on a targeted cognitive ability, and who complete a common set of assessments on the targeted ability and other related clinical, anatomical, and/or performance measures. Such an approach is well-suited for testing cognitive (e.g., IA model) and statistical (e.g., regression) models (Schwartz \& Dell, 2010). It should be noted that case series studies differ from broad patient group studies; patient groups (e.g., a group of individuals with Broca's aphasia) rely on classification schemes that are determined by simple co-occurrences of certain impairments with weak specification of the underlying mechanism(s) for those impairments (Caramazza, 1984). The analysis of case series studies are not as concerned with the mean, or average, of the group, but instead are focused on trends and the variability in those trends (Schwartz \& Dell, 2010). In this way, researchers can identify who deviates from the group (or a sub-group) trend, and explore why their performance differs from those who shared similar characteristics in initial group inclusion (Schwartz \& Dell, 2010). Today, sophisticated regression analyses, like mixed effects models, make it possible to delve deeper into between-subject heterogeneity, even with small sample sizes (e.g., Wiley \& Rapp, 2019). Thus, while heterogeneity is a known factor that cannot, and should not, be eliminated, it is important that model development uses appropriate approaches that account for this heterogeneity. 
Most studies examining networks of words have considered group-level or aggregated networks. Many of the group-level network analyses focus on structural differences in networks which can be used to determine differences between typical and clinical populations. For example, this approach was taken by Castro and Stella (2019; see also Castro et al., 2020) who compared the effect of network measures across different aphasia diagnoses and typical adults to illustrate its ubiquitous effect in word retrieval. However, as noted previously, these broad group-level comparisons miss out on accounting for the heterogeneity between individuals, leading to analysis of "average" networks that may not actually be representative of any individual in the group (Schwartz \& Dell, 2010). As further illustration, Castro et al. (in press) provide evidence of how between-subject heterogeneity in a sample of persons with aphasia contributed to null results for the effect of a network measure shown to influence processing in typical populations. Thus, it is critical that network modeling of word retrieval failures consider a case series approach that allows for correlating network analysis results with performance on other cognitive and language assessments.

To move away from group-level networks requires consideration of individualized networks. This trend towards considering individualized networks has begun in cognitive network science. For example, work by Zemla and Austerweil (2018) estimated semantic networks of individuals and groups of individuals by using a common psycholinguistic task, verbal fluency. In the verbal fluency task, an individual provides as many exemplars of a given category (e.g., animals, words that start with 's') in a specified amount of time. The authors compared a variety of estimation techniques, highlighting their assumptions and limitations (Zemla \& Austerweil, 2018; for a open-source tool to estimate networks, see Zemla et al., 2020). 
While estimating an individual's semantic network would allow for better consideration of between-subject variability, especially when used in a case series approach, there are important cautions to raise with its use in clinical populations. First, estimating individual networks requires repeated data sampling. That is, a single verbal fluency test is not enough to accurately estimate the semantic network of an individual. In the study of aphasia, it is wellknown that repeated sampling is necessary, for example with pre- and post- treatment testing, because performance can vary significantly from day-to-day (and even within day). Thus, although repeated testing does occur in the study of clinical populations, the interpretation of such repeated testing is not identical to that of typical adults. A second concern is the amount of time that lapses between repeated data samplings. In progressive diseases, like semantic dementia, estimating the individual network may be complicated by the actual degradation of the network over time. Furthermore, repeated testing closer in time could be burdensome to the individual, especially if done in conjunction with other cognitive and language assessments (in the spirit of a case series analysis). Therefore, caution must be taken in interpreting and using repeated data samples in the estimation of individual networks of persons with language disorders. The estimation of individual networks is still in its infancy but holds promise for developing better, and more sophisticated, network models of retrieval failures.

\subsection{Progression of disorder/disease: Moving away from static networks}

This last methodological issue recognizes that different clinical language disorders progress in different trajectories over time. For persons with aphasia, there is general agreement that the largest gains in recovery of language abilities occur within 3-6 months of stroke. However, improvement in language abilities can still occur after 6 months post-stroke in what is called the chronic aphasia stage. Many factors contribute to how much improvement occurs for 
those with chronic aphasia, including the severity and type of stroke and how much therapy an individual receives, but more work is still needed in this area to fully understand the varying trajectories of chronic aphasia progression (Johnson et al., 2019; Lazar \& Antoniello, 2008). In progressive diseases, including semantic dementia (Mioshi et al., 2010), there is general agreement that cognitive and language abilities decline over time, often with faster rates of decline in later stages of the disease. Critically, the etiology of the disease impacts exact rates of decline; for example, frontotemporal dementia, which includes semantic dementia, may result in faster decline than Alzheimer's disease (Rascovsky et al., 2005). Critically, the point here is that language and cognitive abilities are not stable in persons with language disorders. While this may also be true of typical adults, the amount of change over time in clinical populations is likely to be more dramatic and consequential to the interpretation and use of clinical data in model development.

In the study of dynamical networks, Castro and Siew (2020) distinguish between questions that focus on the dynamics of a network (i.e., how the network itself changes) and dynamics on a network (i.e., how processes of the system occur). This distinction is important to the development of network models of retrieval failures that use clinical data, such that which dynamical process may need to be manipulated or studied is dependent on the locus of the retrieval failure. In semantic dementia, the dynamical process of interest would be of the network, or how the network structure degrades with the progression of disease. In aphasia, the dynamical process of interest would be on the network, or how the processes occurring in the network change with the progression of aphasia.

While much of the work in cognitive network science has focused on analyzing static network representations, there have also been advances to the study of how networks change 
over time. Early, and continued, work considering the dynamics of a language network have focused on understanding the mechanism for how new words are added to a child's lexicon (e.g., Hills et al., 2009; Siew \& Vitevitch, 2020; Sizemore et al., 2018; Stella et al., 2018; Steyvers \& Tenenbaum, 2005). There has also been a large body of work focused on the degradation of networks with dementia (Bertola et al., 2014; Borge-Holthoefer et al., 2011; Lerner et al., 2009; Zemla \& Austerweil, 2019). Of course, more work is still needed to fully develop a network model of retrieval failures.

There has been less work on how dynamical processes acting on networks change over time. In part, researchers in cognitive network science are still debating what processes act on networks. For example, in the context of search processes in semantic memory (e.g., as required when performing a verbal fluency task), there continues active debate between an optimal foraging and a random walk mechanism (Avery \& Jones, 2018). Optimal foraging emphasizes an important trade-off during search between exploiting a certain part of semantic memory and exploring a novel part of semantic memory (Hills et al., 2012; Jones et al., 2015). Random walks, which resemble spreading activation processes, assume that the structure of the network constrains the search process (Abbott et al., 2015). Regardless of which process mechanism is employed, it is important to remember that these mechanisms may change in function over time in clinical populations. That is, it could be the case that optimal foraging becomes less optimal as dementia progresses, in conjunction with the cognitive impairments that accompany semantic memory degradation. It remains to be seen how dynamical processes acting on networks might also change over time and in the face of a language and/or cognitive impairment. 


\section{Discussion}

This paper described three methodological issues that are critical to the use and interpretation of clinical data, which have implications for continued development of a model of retrieval failures. These issues are accounting for the locus of retrieval failure, heterogeneity of individuals, and progression of disorder/disease. While no model is true, ongoing work must continue in the development and validation of a network model of retrieval failures. This work will require continued interdisciplinary collaboration to ensure that the model is not just theoretically but also clinically valid, as has been done in the development of prominent language models, like Dell's Interactive Activation model. Researchers active in the development of a network model of retrieval failures need to ensure that the model is theoretically well-informed, which requires accounting for the methodological issues described, as the model could potentially impact the lives of individuals seeking treatments that are based on knowledge derived from the model. With that said, such a network model of retrieval failures has significant potential to address long-standing questions regarding the influence of structure on process, as well as provide novel directions for the development of clinical tools and treatments. 


\section{References}

Abbott, J. T., Austerweil, J. L., \& Griffiths, T. L. (2015). Random Walks on Semantic Networks Can Resemble Optimal Foraging. Psychological Review, 122(3), 558-569.

Avery, J., \& Jones, M. (2018). Comparing models of semantic fluency: Do humans forage optimally or walk randomly? In T. T. Rogers, M. Rau, X. Zhu, C. W. Kalish (Eds.), Proceedings of the 40th Annual Meeting of the Cognitive Science Society (pp. 118-123). Cognitive Science Society.

Baronchelli, A., Ferrer-i-Cancho, R., Pastor-Satorras, R., Chater, N., \& Christiansen, M. H. (2013). Networks in Cognitive Science. Trends in Cognitive Sciences, 17(7), 348-360.

Beckage, N. M., \& Colunga, E. (2015). Language networks as models of cognition:

Understanding cognition through language. In A. Mehler, A. Lucking, S. Banisch, P. Blanchard, \& B. Job (Eds.), Towards a Theoretical Framework for Analyzing Complex Linguistic Networks (pp. 3-28). Springer.

Beeson, P. M., Holland, A. L., \& Murray, L. L. (1997). Naming famous people: An examination of tip-of-the-tongue phenomena in aphasia and Alzheimer's disease Naming famous people. Aphasiology, 11(415), 323-336.

Bertola, L., Mota, N. B., Copelli, M., Rivero, T., Diniz, B. S., Romano-Silva, M. A, Ribeiro, S., \& Malloy-Diniz, L. F. (2014). Graph analysis of verbal fluency test discriminate between patients with Alzheimer's disease, mild cognitive impairment and normal elderly controls. Frontiers in Aging Neuroscience, 6, 185.

Borge-Holthoefer, J., Moreno, Y., \& Arenas, A. (2011). Modeling abnormal priming in Alzheimer's patients with a free association network. PLoS ONE, 6(8), e22651.

Burke, D., MacKay, D., Worthley, J., \& Wade, E. (1991). On the tip of the tongue: What causes 
word finding failures in young and older adults? Journal of Memory and Language, 30, $542-579$.

Caramazza, A. (1984). The Logic of Neuropsychoiogical Research and the Problem of Patient Classification in Aphasia. Brain and Language, 21(1), 9-20.

Castro, N., Nadeau, S. E., \& Kendall, D. L. (in press). The challenge of achieving greater generalization in phonological treatment of aphasia. Aphasiology.

Castro, N., \& Siew, C. S. Q. (2020). Contributions of modern network science to the cognitive sciences: Revisiting research spirals of representation and process. Proceedings of the Royal Society A: Mathematical, Physical and Engineering Sciences, 476(2238), 20190825.

Castro, N., \& Stella, M. (2019). The multiplex structure of the mental lexicon influences picture naming in people with aphasia. Journal of Complex Networks, 7(6), 913-931.

Castro, N., Stella, M., \& Siew, C. (2020). Quantifying the interplay of semantics and phonology during failures of word retrieval by people with aphasia using a multiplex lexical network. Cognitive Science, 44(9), e12881.

Chan, K., \& Vitevitch, M. (2010). Network structure influences speech production. Cognitive Science, 34(4), 685-697.

Clough, S., \& Gordon, J. K. (2020). Fluent or nonfluent? Part A. Underlying contributors to categorical classifications of fluency in aphasia. Aphasiology, 34(5), 515-539.

Dell, G. (1986). A Spreading-Activation Theory of Retrieval in Sentence Production. Psychological Review, 93(3), 283-321.

Dell, G., \& O’Seaghdha, P. (1992). Stages of Lexical Access in Language Production. Cognition, 42, 287-314.

Dell, G., Schwartz, M., Martin, N., Saffran, E., \& Gagnon, D. (1997). Lexical Access in Aphasic 
and Nonaphasic Speakers. Psychological Review, 104(4), 801-838.

Dignam, J., Copland, D., O’Brien, K., Burfein, P., Khan, A., \& Rodriguez, A. D. (2017). Influence of cognitive ability on therapy outcomes for anomia in adults with chronic poststroke aphasia. Journal of Speech, Language, and Hearing Research, 60(2), 406-421.

Foygel, D., \& Dell, G. (2000). Models of Impaired Lexical Access in Speech Production. Journal of Memory and Language, 43(2), 182-216.

Gordon, J. (2002). Phonological neighborhood effects in aphasic speech errors: Spontaneous and structured contexts. Brain and Language, 82(2), 113-145.

Henry, M. L., Beeson, P. M., \& Rapcsak, S. Z. (2008). Treatment for anomia in semantic dementia. Seminars in Speech and Language, 29(1), 60-70.

Hills, T., Jones, M., \& Todd, P. (2012). Optimal foraging in semantic memory. Psychological Review, 119(2), 431-440.

Hills, T., Maouene, M., Maouene, J., Sheya, A., \& Smith, L. (2009). Longitudinal analysis of early semantic networks: preferential attachment or preferential acquisition? Psychological Science, 20(6), 729-739.

Johnson, L., Basilakos, A., Yourganov, G., Cai, B., Bonilha, L., Rorden, C., \& Fridriksson, J. (2019). Progression of aphasia severity in the chronic stages of stroke. American Journal of Speech-Language Pathology, 28(2), 639-649.

Jones, M., Hills, T., \& Todd, P. (2015). Hidden processes in structural representations: A reply to Abbott, Austerweil, and Griffiths (2015). Psychological Review, 122(3), 570-574.

Kenett, Y., \& Faust, M. (2019). Clinical cognitive networks: A graph theory approach. In M. Vitevitch (Ed.), Network Science in Cognitive Psychology (pp. 136-165). Routledge.

Lazar, R. M., \& Antoniello, D. (2008). Variability in Recovery from Aphasia. Current 
Neurology and Neuroscience Reports, 8, 497-502.

Lerner, A., Ogrocki, P., \& Thomas, P. (2009). Network graph analysis of category fluency testing. Cognitive and Behavioral Neurology, 22(1), 45-52.

Levelt, W. (1999). Models of word production. Trends in Cognitive Sciences, 3(6), 223-232.

Martin, N., \& Dell, G. (2019). Maintenance versus transmission deficits: The effect of delay on naming performance in aphasia. Frontiers in Human Neuroscience, 13, 406.

McNeil, M. (1982). The nature of aphasia in adults. In N. Lass, L. McReynolds, J. Northern, \& D. Yoder (Eds.), Speech, Language, and Hearing: Vol III. Pathologies of Speech and Language (pp. 692-740). W.B. Saunders.

McNeil, M., \& Pratt, S. (2001). Defining aphasia: Some theoretical and clinical implications of operating from a formal definition. Taylor \& Francis, 15(10-11), 901-911.

Middleton, E., \& Schwartz, M. (2010). Density pervades: An analysis of phonological neighbourhood density effects in aphasic speakers with different types of naming impairment. Cognitive Neuropsychology, 27(5), 401-427.

Mioshi, E., Hsieh, S., Savage, S., Hornberger, M., \& Hodges, J. (2010). Clinical staging and disease progression in frontotemporal dementia. Neurology, 74(20), 1591-1597.

Mirman, D., \& Britt, A. (2014). What we talk about when we talk about access deficits. Philosophical Transactions of the Royal Society B: Biological Sciences, 369(1634), 20120388.

Mirman, D., \& Magnuson, J. (2008). Attractor dynamics and semantic neighborhood density: processing is slowed by near neighbors and speeded by distant neighbors. Journal of Experimental Psychology. Learning, Memory, and Cognition, 34(1), 65-79.

Rascovsky, K., Salmon, D. P., Lipton, A. M., Leverenz, J. B., DeCarli, C., Jagust, W. J., Clark, 
C. M., Mendez, M.. F., Tang-Wai, D. F., Graff-Radford, N. R., \& Galasko, D. (2005). Rate of progression differs in frontotemporal dementia and Alzheimer disease. Neurology, 65(3), $397-403$.

Schwartz, M., \& Dell, G. (2010). Case series investigations in cognitive neuropsychology. Cognitive Neuropsychology, 27(6), 477-494.

Schwartz, M., Dell, G., Martin, N., Gahl, S., \& Sobel, P. (2006). A case-series test of the interactive two-step model of lexical access: Evidence from picture naming. Journal of Memory and Language, 54(2), 228-264.

Shafto, M., Burke, D., \& Stamatakis, E. (2007). On the tip-of-the-tongue: neural correlates of increased word-finding failures in normal aging. Journal of Cognitive Neuroscience, 19(12), 2060-2070.

Siew, C. (2019). spreadr: A R package to simulate spreading activation in a network. Behavior Research Methods, 51(2), 910-929.

Siew, C., \& Vitevitch, M. (2020). An Investigation of Network Growth Principles in the Phonological Language Network. Journal of Experimental Psychology: General, 149(12), 2376-2394.

Siew, C., Wulff, D., Beckage, N., \& Kenett, Y. (2019). Cognitive Network Science: A review of research on cognition through the lens of network representations, processes, and dynamics. Complexity, 2019, 2108423.

Sizemore, A., Karuza, E., Giusti, C., \& Bassett, D. (2018). Knowledge gaps in the early growth of semantic feature networks. Nature Human Behaviour, 2(9), 682-692.

Stella, M., Beckage, N., Brede, M., \& De Domenico, M. (2018). Multiplex model of mental lexicon reveals explosive learning in humans. Scientific Reports, 8, 2259. 
Steyvers, M., \& Tenenbaum, J. B. (2005). The large-scale structure of semantic networks: statistical analyses and a model of semantic growth. Cognitive Science, 29(1), 41-78.

Tremblay, P., \& Dick, A. (2016). Broca and Wernicke are dead, or moving past the classic model of language neurobiology. Brain and Language, 162, 60-71.

Vitevitch, M. (2008). What can graph theory tell us about word learning and lexical retrieval? Journal of Speech, Language, and Hearing Research, 51, 408-422.

Vitevitch, M., \& Castro, N. (2015). Using network science in the language sciences and clinic. International Journal of Speech-Language Pathology, 17(1), 13-25.

Vitevitch, M., Chan, K., \& Goldstein, R. (2014). Insights into failed lexical retrieval from network science. Cognitive Psychology, 68, 1-32.

Vitevitch, M., Ercal, G., \& Adagarla, B. (2011). Simulating retrieval from a highly clustered network: Implications for spoken word recognition. Frontiers in Psychology, 2, 369.

Vitevitch, M., Goldstein, R., Siew, C., \& Castro, N. (2014). Using complex networks to understand the mental lexicon. Yearbook of the Poznan Linguistic Meeting, 1, 119-138.

Vitevitch, M., \& Luce, P. (2016). Phonological Neighborhood Effects in Spoken Word Perception and Production. Annual Review of Linguistics, 2, 75-94.

Vitevitch, M., \& Sommers, M. (2003). The facilitative influence of phonological similarity and neighborhood frequency in speech production in younger and older adults. Memory \& Cognition, 31(4), 491-504.

Vitevitch, M., \& Storkel, H. (2013). Examining the Acquisition of Phonological Word Forms with Computational Experiments. Language and Speech, 56(4), 493-527.

Wiley, R. W., \& Rapp, B. (2019). Statistical analysis in Small-N Designs: using linear mixedeffects modeling for evaluating intervention effectiveness. Aphasiology, 33(1), 1-30. 
Zemla, J., \& Austerweil, J. (2018). Estimating semantic networks of groups and individuals from fluency data. Computational Brain \& Behavior, 1(1), 36-58.

Zemla, J., \& Austerweil, J. (2019). Analyzing knowledge retrieval impairments associated with Alzheimer’s disease using network analyses. Complexity, 2019, 4203158.

Zemla, J., Cao, K., Mueller, K., \& Austerweil, J. (2020). SNAFU: The Semantic Network and Fluency Utility. Behavior Research Methods, 52, 1681-1699. 


\section{Figure 1}

Structure of the Interactive Activation Model and Networks of Words.

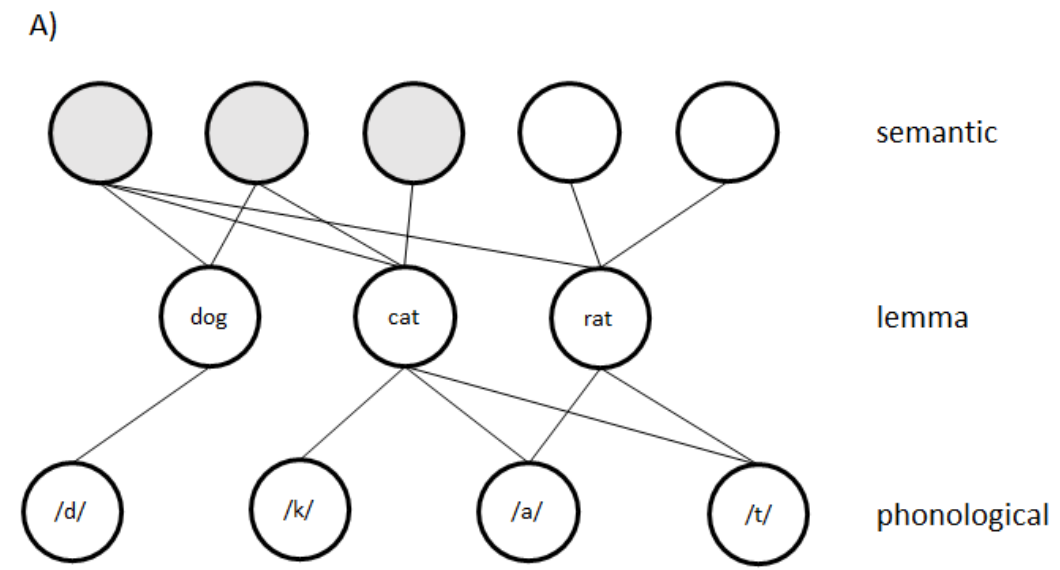

B)
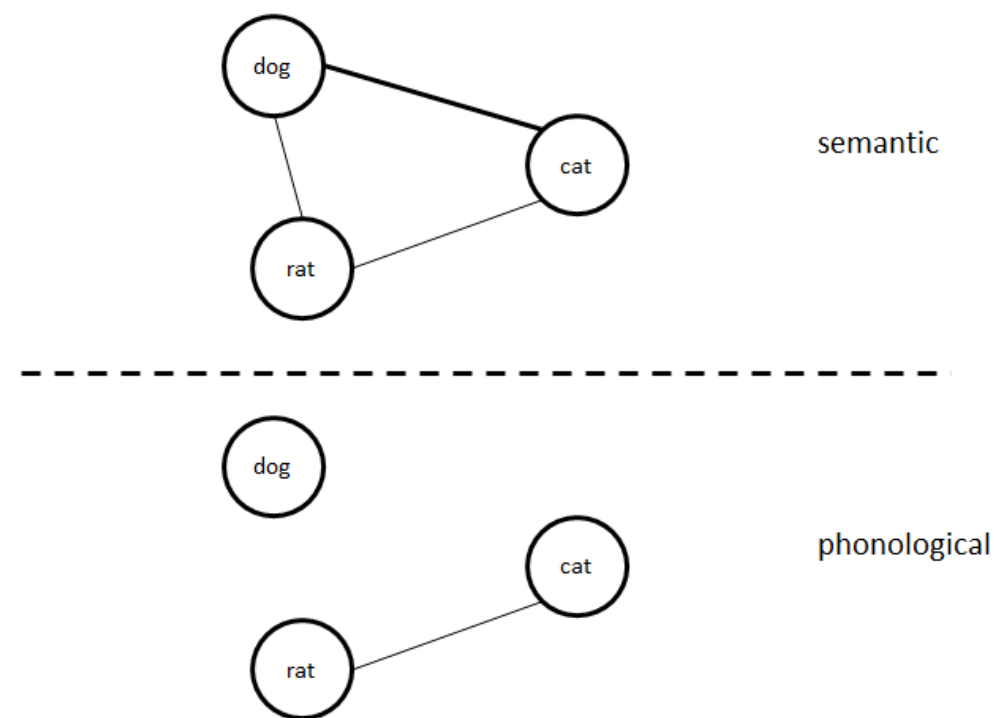

Note. A: A schematic of the Interactive Activation model (Dell, 1986; Dell \& O'Seaghdha, 1992) depicting the three systems of nodes: semantic, lemma, and phonological. The grayed semantic nodes reflect features of a to-be-named picture. B: A schematic of Networks of Words, the top representing semantic similarity and the bottom representing phonological similarity. The bolded connection between dog and cat in the top network reflects greater semantic similarity. 